\title{
Graphene and Carbon Nanotube Sensors of THz field
}

\author{
Serhii E. Shafraniuk \\ Physics and Astronomy Department, Northwestern University, 2145 Sheridan Rd., Evanston IL 60208, \\ USA \\ s-shafraniuk@northwestern.edu
}

\begin{abstract}
The THz field quantum dot sensors (T-sensors) made of graphene and carbon nanotubes have a very low detecting power, tiny dimensions, and they are tunable dynamically. The sensors simultaneously measure the $\mathrm{THz}$ field intensity, polarization, and spectrum. The graphene and carbon nanotube sensors exploit a change in the intrinsic transport properties of the quantum dot under influence of the photon-assisted resonant tunneling.
\end{abstract}

Key words: carbon, nanotube, quantum dot, THz field, sensors.

\section{Introduction}

A strong demand for sensors working in the $\mathrm{THz}$ domain which covers the electromagnetic field spectrum [1] emerges in various areas of human activity, including medicine, space research, defense and security. There are many other aspects why the T-rays are interesting [2-5]. In particular, many chemical inorganic and organic molecules have oscillation and rotation resonances occurring in the $\mathrm{THz}$ frequency region. Each chemical analyte is characterized by a unique set of spectral lines which might serve as fingerprints for their identification. The measuring the reflection and transmission of the $\mathrm{THz}$ waves allows remote and instant sensing of the corresponding chemicals. Therefore the $\mathrm{THz}$ field sensors are capable to remote and instant disclosing, identification, and chemical analysis of many analytes. Another important feature of T-rays is the sub-millimeter wave length which is comparable to the thickness of human closing and plastic covers. It causes the T-rays to be easily penetrated through thin layers of closes or covers which reflect the conventional sunlight the other way. Thus the $\mathrm{THz}$ spectral analyzer might detect the concealed weapon, hidden explosives, or toxic chemicals. That capability of the T-sensors is exploited in defense and security applications [1]. In our work we pay attention to the quantum dot (QD) carbon nanotube and graphene sensors where an external a.c. field induces electron transitions between quantized levels as illustrated in Figures 1 and 2. [6]

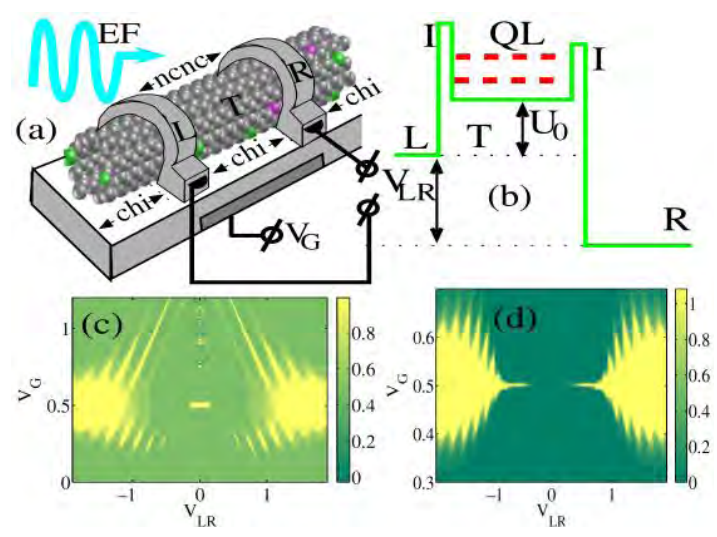

Fig. $1 . \quad$ a) Design of a carbon nanotube (CNT) field effect transistor (FET) where $L$ and $R$ are metallic electrodes. (b) Schematics of a quantum well with localized levels QL (shown in red). c) and d) are the contour plots of the differential conductivity through the dot.

\section{Basis of the THz Detecting}

An immediate detecting of the $\mathrm{THz}$ waves by quantum well devices however represents a non-trivial task. On one hand, a fundamental difficulty is that the energy $h f$ of a T-ray photon with frequency $f$ ( $h$ is the Plank constant) is relatively low. On the other hand, a satisfactory T-ray frequency resolution requires that $h v=\Delta E \geq k T^{*}$ ( $\Delta E$ is the quantized electron energy spacing) must exceed the electron temperature $T^{\star}$ in the dot, which for frequency $f$ $=\Delta E / h=1 \mathrm{THz}$ is about $50 \mathrm{~K}$. For such reasons, to achieve a good spectral resolution, the quantum dot must be refrigerated well below $50 \mathrm{~K}$. Besides, the T-ray sensor elements are supposed to being exceptionally sensitive to the low-energy photons. One more 
complications is that the T-rays are not reflected by conventional mirrors nor they are focused by conventional lenses. Absence of appropriate T-ray mirrors and lenses makes the control of T-rays problematic. Thus creating and development of the T-ray optical devices represents another tremendous task.

\section{Experimental Approach}

Using of the novel material and the nanotechnology are the key elements which are able to handling the aforementioned problems. [8-12] Most important components of our QD carbon nanotube (CNT) and graphene $(\mathrm{G}) \mathrm{T}$ sensor is either a section of single-wall CNT by diameter $d=2-3 \mathrm{~nm}$ and by length $L=200-800$ $\mathrm{nm}$ or a graphene stripe by width $6-12 \mathrm{~nm}$ and the same length. One new fundamental feature of those materials is that the electrons in $\mathrm{G}$ and CNT behave as relativistic particles. This makes them different from electrons in ordinary semiconductors where they behave as spinless excitations. In contrast to the latter, the electrons in the new carbon materials are characterized by chirality and by the two 1/2pseudospins. This impacts the whole scenario of the electron transport in carbon materials and also causes a number of controversies. In particular, the pseudospins conserve during the electron scattering on impurities, on phonons, and on other electrons. Hence the electron movement in the CNT/G devices is more ballistic than diffusive. The electron mean free path in the carbon nanotube/graphene devices is determined by scatterings of an electron on other electrons, and also on phonons and impurities. The long electron mean free path $I_{e}$ $\left(l_{e}=\min \left\{l_{e e}, l_{e p}, l_{e i}\right\}, \quad\right.$ where $l_{e e, e p, e i}=v \tau_{e e, e p, e i}$, and $\tau_{e e, e p, e i}$ are scattering times of an electron on the other electrons, phonons, and impurities) which typically exceeds the nanotube section length actually means that the nanotube/graphene is intrinsically "purified". Intrinsic "puriness" of the carbon nanotube/graphene devices originates from the relativistic nature of the electrons. The ballistic propagation of quasiparticle excitations in CNT/G comes in contrast to the electron transport in regular semiconductors. A difference between the CNT/G and a conventional $2 \mathrm{D}$ semiconductor is that a strong electron-electron scattering impedes the ballistic transport. [8-12]

Good quantized levels (QL) inside the graphene stripe or in the CNT section (see left panel in Figure 2a,b) are formed because the electron mean free path is long there. The quantized energy level's position and width inside the CNT quantum dot are adjusted by applying of appropriate electric potentials. This is accomplished by attaching of two or more external source and drain electrodes to the CNT section where the quantized states emerge. [2-6] By applying the external source drain $V_{L R}$ and gate $V_{G}$ voltages one initializes the electron transport through the dot. The electrons also interact with the external $\mathrm{THz}$ field (EF) which induces the photon-assisted tunneling across the quantum dot. Because the tunneling a.c. current affects the electron quantization conditions, it also modifies the quantized states.

The changes in the system induced by the a.c. field are detected by measuring the quantum dot conductance. The junction's conductance is then altered by several orders of magnitude against its steady state value.

\section{CNT and Graphene Quantum Dots for THz Sensing}

Strong response of the electron transport to the external electromagnetic field is exploited in the new highly sensitive $\mathrm{THz}$ detectors as schematically shown in Figures 1 and 2. The QD detectors utilize a change in the electron transport properties under influence of a.c. external fields. If the electron energy in the source and drain electrodes matches the quantized level position $E_{n}$, providing $h f=E_{1}-E_{0}$ (where $h$ is Plank constant, $f$ is the THz field frequency, $E_{0}$ and $E_{1}$ are the energies of the lowest and 1st excited quantized energy levels), the tunneling probability through the QD is high. Corresponding resonant peaks in the d.c. conductance $G$ are measured across QD experimentally (see right panel in Figure 1). An external a.c. field changes the electron energy during the tunneling by the amount of photon energies $n h f \quad(n$ is the number of absorbed/emitted photons, hf is the photon energy). Consequently, the energy matching condition is changing as $h f=E_{1}-E_{0}=>n h f=$ $E_{1}-E_{0}$. The a.c. field modified resonance condition ensures that the side peaks in the d.c. conductance arise at new values $V_{S D}=E_{1}-n h f$ and $V_{S D}=E_{1}+n h f$ of the source-drain voltage $V_{\mathrm{SD}}$. At low temperatures, the side peak amplitude increases by a few orders of magnitude as compared to its steady state value. The sharp changes in the QD conductance constitute an idea of the a.c. field sensing. The sharp and crisp resonances ensure the high accuracy of the QD $\mathrm{THz}$ detectors. By measuring the resonance width, spacing, and magnitude, one immediately determines the frequency, intensity, and even the polarization of external $\mathrm{THz}$ field. Another important issue is reducing of the intrinsic and 
parasitic noises which are limiting the sensitivity of the device. To reduce the thermal noises in the device, we have implemented so-called intrinsic cooling the carbon nanotube quantum well $\mathrm{THz}$ sensor the idea of which has been illustrated by the right panel in Figure 2.

\section{The Sensing Principle}

The $\mathrm{THz}$ sensing principles are based on unconventional intrinsic properties of graphene and carbon nanotubes which involve so-called

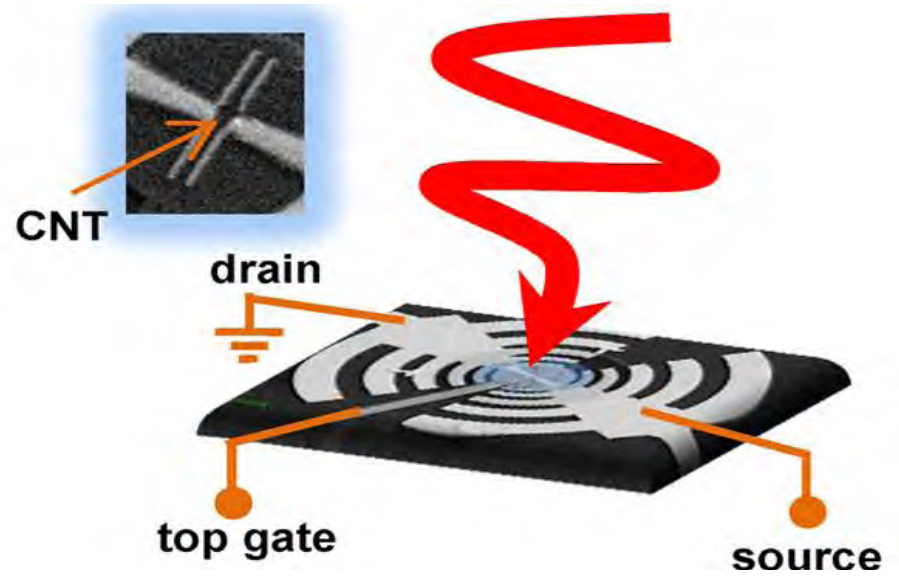

chiral transport. The honeycomb crystal symmetry of graphene and carbon nanotubes determines the wave function symmetry of the elementary excitations in that system.

The $1 / 2$-pseudospin is conserved during the tunneling, so it provides additional quantum mechanical selection rules regarded as the $1 / 2$ pseudospin conservation law.

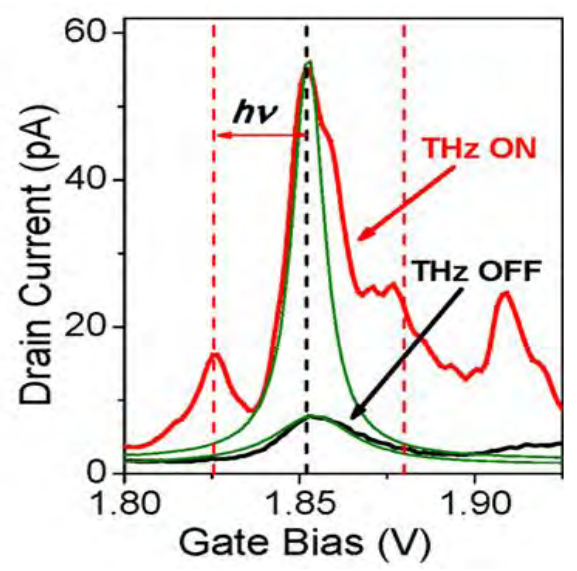

Fig. 2. Left: Experimental setup for THz sensing with a carbon nanotube field effect transistor. Right: The I-V curves of the THz sensor with the a.c. field off (black curve) and on (red curve). The green curve is the Lorentzian fitting which suggests that the Coulomb peak width is reduced when the $\mathrm{THz}$ field is on.

For such reasons, the quasiparticle excitations inside the CNT/G section are not just merely conventional electrons or holes as they are in regular semiconductors or metals. [2-6] The charge carriers actually behave as chiral particles with two $1 / 2$-spins per each particle. [912]

\section{Role of chirality in graphene}

The directions "up" and "down " of one $1 / 2$ pseudospin are attributed to two different spectral branches. They correspond to electron (or hole) excitations with either positive $(+V)$ or negative $(-V)$ electron velocity per each. The two "up" and "down " directions of another $1 / 2$ pseudospin are related to the $\mathrm{K}$ (or K') point correspondingly.

The pseudospin conservation laws outline basic transport properties of the $G$ and CNT. Besides, those additional conservation laws make the transport properties of $G$ and CNT being distinct from properties of regular conductors. One important consequence of the new conservation laws is that the rates of electron-impurity and electron-phonon collisions are much lower than in regular semiconductors and metals. The pseudospin conservation insures that the electron transport in $\mathrm{G}$ and/or CNT remains ballistic up to the room temperature. It is an immediate outcome of the phase-coherent propagation of the chiral particles. [9-12] The difference between the chiral and non-chiral transport is illustrated by the contour plots (see Figures 2c,d). Contour plots in Figures 2c,d suggest that the chirality plays an important role in functioning the CNT and graphene devices.

Our T-sensor model describes the quantum dot (QD) which is formed either by a graphene stripe or by a section of single wall CNT resting on (or suspended over) a dielectric substrate with electrodes attached (see Figures 1, 2). Interference between incident electron waves with the waves backward reflected from the Schottky barriers causes the electron motion inside the CNT section to be quantized. The gate electrode is placed underneath of the dielectric $\mathrm{SiO}_{2}$ substrate as shown in left panels of Figs. 1, 2. In this way, the quantum dot is formed inside the CNT/G section enclosed between the two Schottky barriers. The whole setup represents a field effect transistor where the metallic electrodes $L$ and $R$ serve as source and drain. The gate voltage $V_{G}$ controls the quantized level (red dash in Fig. 2b) positions while injecting of electrons from the $L$ and $R$ electrodes into the CNT/G changes population of the levels. The CNT (graphene) QD is exposed to the external $\mathrm{THz}$ field EF. Left panel in Figure 2 shows the photo while illustration of the working principle is sketched in Figure 3. The carbon nanotube section shown in Figures 
1,2 has typical length $L=250-5000 \mathrm{~nm}$ and is enclosed between two metallic electrodes $L$ and $\mathrm{R}$ deposited on the top of the CNT. The CNT section length is selected to get the quantized level spacing $\sim 4 \mathrm{mV}$ which corresponds to about $1 \mathrm{THz}$.

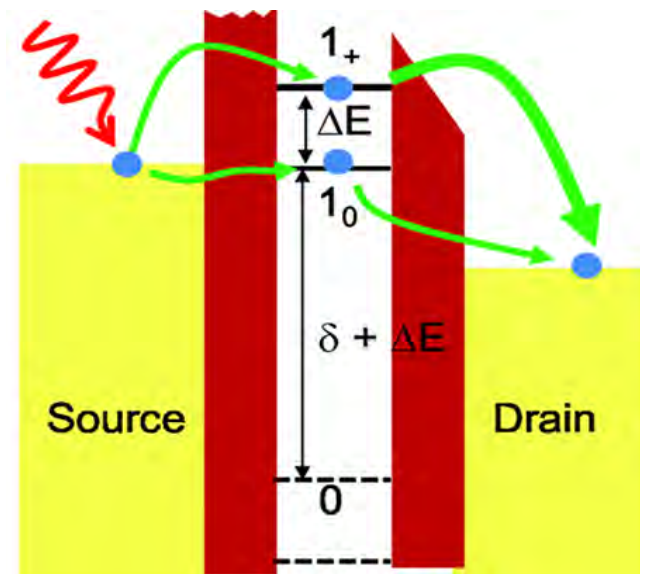

Fig. 3. Principle of the $\mathrm{THz}$ field induced cooling of the quantum dot. It the field frequency less than the level spacing, an electron escapes from the dot to the drain electrode slower (thin green arrow on the right) than from the upper level (thick green arrow on the right).

Since concentrations of the electric charge carriers in the metallic electrodes and in the CNT section differ, the interface Schottky barriers arise. The barriers are located right at the interfaces between the metallic electrodes and the CNT (see inset in Figure 2a,b). Because the 1/2-pseudospin conserves during electron scattering on the lattice defects, phonons, and other electrons, the electron propagation inside the CNT is ballistic. The $\mathrm{THz}$ field photons are absorbed by the tunneling electrons. [9-12] Relationship between the electric current and the applied a.c. field depends on the source - drain and gate dc voltages, and it also changes versus the a.c. field amplitude, frequency, and polarization. In a most trivial case, [9-12] the external a.c. electric field vector is aligned with the carbon nanotube or the graphene stripe axis. Then the a.c. voltage effectively shifts the source drain voltage as $V_{G} \rightarrow V_{G}+n h f$. That case corresponds to a setup where the external $\mathrm{THz}$ field is polarized in-perpendicular to the CNT section.

The $\mathrm{THz}$ field induces an a.c. voltage which adds to the electron electrochemical potential inside the CNT section. It can also be understood as the a.c. field causes an additional time dependence of the gate voltage $\mathrm{V}_{\mathrm{G}}$ [9-12]

The suggested model of the photon-assisted chiral tunneling (Shafraniuk, 2009, 2011) can be useful to interpreting of existing experimental data. [2-6] The model is capable of addressing the junctions of arbitrary transparency under influence of a.c. fields having arbitrary amplitude and frequency.
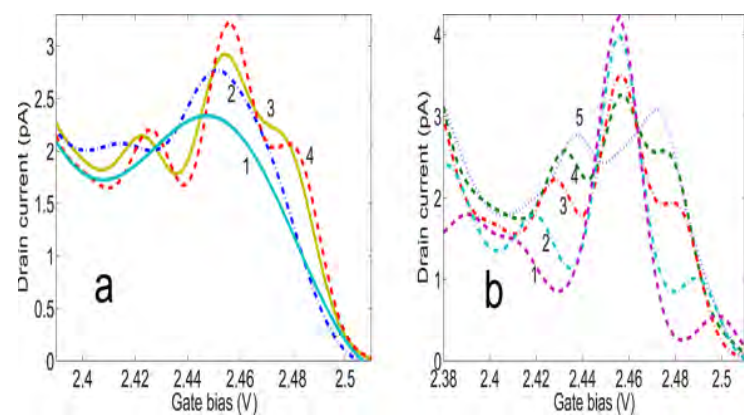

Fig. 4. Numeric fitting of the obtained experimental data. The results indicate splitting of the main Coulomb peak into the three peaks (one main and two satellites) when the $\mathrm{THz}$ field is applied. According to the theory [9-12] the spacing between the main and the satellite peaks is $\Delta V=h f / e$.

Fabrication and experimental study of the carbon nanotube quantum dot $\mathrm{THz}$ sensors (CNT-Dot) had been reported in the recent papers. [2-6] Basic idea is to multiply two different resonances: (i) A geometrical quantization of the electron motion, and (ii) The discreteness of the electron charge. The CNTDot device had been fabricated using a small section of CNT which has connected two weakly-coupled source (L) and drain (R) electrodes (see left panel of Figure 2). Besides, the dot had been coupled to a third gate electrode capacitively. Because of a very low value of the total capacitance, $C$, the charging energy $U$ of the CNT section was big enough to affect the whole electron transport through the dot. The charging effects were noticeable as sharp peaks in the source-drain conductance as a function of gate voltage at temperatures $T$ $<U$. The peak series has correspond to resonant elastic tunneling of single electrons through the dot. The spacing between the peaks is a sum of the energy level spacing $E_{1}$ $E_{0}$ and the charging energy of the dot $U$. An external $\mathrm{THz}$ field causes an additional quantization of the electron states: Besides the geometrical quantization inside the CNT-Dot, and the quantization of the electron charge, the $\mathrm{THz}$ field of frequency $\mathrm{f}$ changes the electron energy by amount $n h f$ where $n$ is the integer number. The latter energy change is related to absorption/emission of $n$ photons during the single electron tunneling through the dot. Such a photon-assisted single-electron tunneling, PASET, (inelastic tunneling with absorption and emission of $\mathrm{THz}$ photons) is visible as satellite peaks in the dot's d.c. differential conductance. 
The PASET magnitude is determined by the field intensity and voltage spacing between the main and satellite peaks which is proportional to the photon energy. [7] This makes a difference from the conventional bolometric sensors, since the PASET sensors can determine both the intensity and the frequency of the radiation. A strong advantage of the CNT-Dot detector is that it operates as a wide band spectrometer from a few hundred $\mathrm{GHz}$ up to frequencies corresponding to photon energy $h f$, which is on the order of $10 \mathrm{meV}(2.5 \mathrm{THz})$ or larger.

A successful detecting of a weak $\mathrm{THz}$ field had been recently accomplished by Kawano et al. [2-5] They implemented the aforementioned mechanism of photon assisted tunneling, while the coupling power of $\mathrm{THz}$ field to the CNT-Dot was in the femtowatt range. The initial experiments [2-5] had revealed also basic issues that hindered further research. They are (a) coupling between the detector and the 10 $\mathrm{mW}$ laser source was too weak, and (b) a difficulty to reproducing of the results on multiple samples.

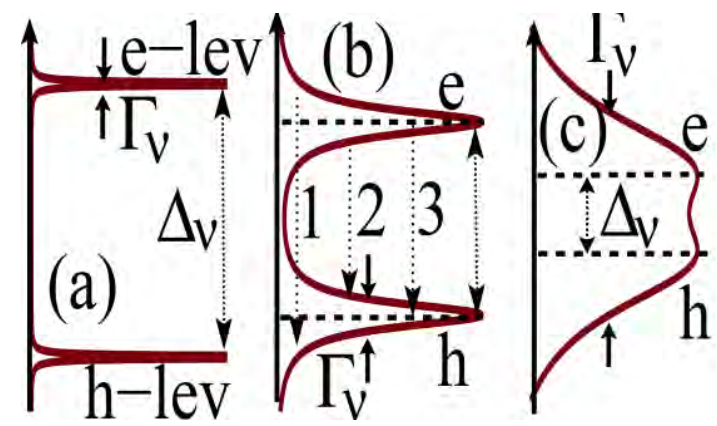

Fig. 5. The the a.c. field induced inter level transitions versus splitting $\Delta_{v}$ between two energy levels and their width $\Gamma_{v}$.

More advanced approach [6] involves a novel design with a local top gate for each device. Then the $\mathrm{THz}$ radiation is well coupled to CNTDots through broad band on-chip antennas. Simultaneously, the antenna electrodes had been also used as source and drain electrodes. The suggested design, [6] ensures considerable response to much weaker sources (power smaller than $10 \mathrm{~W}$ ) within a broad band. The improved response of the CNT-Dot to the $\mathrm{THz}$ field allows observing of new features which had not been formerly visible when the coupling was too weak. They constitute a crucial difference between our sensor and a similar CNT-Dot device studied earlier. (Kawano et al.) Namely, in addition to the satellite peaks on the left and right side of the main peaks, the authors [6] detected an anomalous increase and sharpening of the main peak (see right panel in Figure 1). That increase leads to a conclusion that the effective electron temperature is decreased by the $\mathrm{THz}$ irradiation, which is counterintuitive to general expectations since it occurs due to a nonequilibrium cooling effect. The latter effect is responsible for extracting of excited "hot" electrons from the quantum dot into the drain $R$ electrode. It results in an efficient field-induced cooling of our CNT-Dot sensor when the THz field is on (see Figure 1, right panel).

Unfortunately, in the experiment [6], the SET peak width was comparable to the photon energy $h f$ which was too wide to resolve the satellite PASET peaks. Since the satellite peak spacing was about the same as the central PASET peak width, all the three peaks (i.e., one central peak and the two satellite peaks) are merged altogether into a single wider peak. Along with the peak broadening, there was a strong enhancement of the main peak, especially at frequencies above $800 \mathrm{GHz}$, corresponding to photon energy larger than the level spacing for this device. The aforesaid suggests that the desirable peak separation can be achieved when implementing devices with narrower Coulomb peaks. The narrower Coulomb peaks had been obtained for device $B$, which allowed the PASET satellite peaks to be clearly distinguished (see Figure 1). The behavior of the satellite peak positions versus the $\mathrm{THz}$ field intensity and frequency shown in Figure 1 is well consistent with the photon assisted tunneling concept. Namely, the side peak height increases when the $\mathrm{THz}$ field is stronger while the spacing between the side peaks and the main peaks increases proportionally to $h f$.

The unconventional tendency which had been observed in the experiment [6] become explicable when considering non-equilibrium effects which complement the orthodox PASET model. [7] Those non-equilibrium effects in the CNT-Dot are described by the quantum kinetic equation. The quantum kinetic equation allows computing of the non-equilibrium electron distribution function. In accordance with the standard quantum dot theory [7] we assume that the tunneling rates $\Gamma$ through source-dot and dot-drain Schottky barriers are increasing versus the tunneling electron energy.

The computation results for the drain current versus gate voltage, with different field intensities and frequencies are presented in Figure 4. The obtained curves show distinct satellite peaks, with spacing from the main peak proportional to the photon energy hf. The theoretical results are well consistent with the experimental data. One can see that the computed drain current curves indicate an anomalous increase of the main peak when 
either the field intensity or its frequency $f$ are increased in agreement with the experiment. For calculations we assumed that the lattice temperature on the dot being the same as for the source and drain electrodes, $4 \mathrm{~K}$. Then using it as a boundary condition, we compute the effective electron temperature $T^{*}$ in the dot. The effective temperature $T^{*}$ is obtained by balancing the energy gain of the dot supplied by the $\mathrm{THz}$ field on one hand and the energy taken out by escaping from the dot electrons on the other hand. The escaping into the source and drain electrodes electrons drain the dot energy down causing $T^{*}$ to decrease as the $\mathrm{THz}$ frequency grows.

A simple physical explanation of this non-trivial cooling is given in right panel of Figure 2. The cooling has a non-equilibrium origin and emerges when the $\mathrm{THz}$ frequency is sufficiently high. When the field frequency is too low, $f<$ $\left(E_{1}-E_{0}\right) / h$, the electrons populate mainly level $1_{0}$ located at energy $E_{0}$ because the a.c. field cannot excite them to the upper $1_{+}$level which energy is $E_{1}+U$. The upper $1_{+}$level becomes populated only if $h f>E_{1}-E_{0}+U$. A key factor which determines the cooling efficiency is that the electron lifetime on the lower level $1_{0}$ is much longer than on the upper level $1_{+}$. That happens because the tunneling rate $\Gamma$ increases when the electron energy is $E_{1}+U$, then $h f>E_{1}-E_{0}+U$, and the electrons escape from the level $1_{+}$to the drain electrode $R$ much faster than they did from $1_{0}$ (as indicated by the thicker green arrow for $1_{+}$in Figure 2). Since the "hottest" electrons escape at a higher rate when $h f>E_{1}-E_{0}+U$, the effective electron temperature $T^{*}$ in the dot decreases as soon as the $\mathrm{THz}$ field is applied. It results in the main peak height to increase and while the peak narrows, as illustrated by right panel on Figures 1.

The above experimental data suggest that CNT-Dots are extremely sensitive quantum detectors and potential building blocks for $\mathrm{THz}$ (sub-millimeter) spectrometers. The nonequilibrium cooling has a great potential to novel approaches for higher temperature operation, which is highly desirable for practical applications.

\section{References}

[1] S. Shafraniuk, Nanosensors of External Fields. In H. S. Nalwa, eds., Encyclopedia of Nanoscience and Nanotechnology. American Scientific Publishers 18, 413-454 (2011).

[2] T. Fuse, Y. Kawano, T. Yamaguchi, Y. Aoyagi, and K. Ishibashi, Quantum response of carbon nanotube quantum dots to terahertz wave irradiation, Nanotechnology 18, 044001 (2007); doi:10.1088/0957-4484/18/4/044001.

[3] Y. Kawano, T. Fuse, S. Toyokawa, S. Uchida, K. Ishibashi. Terahertz photon-assisted tunneling in carbon nanotube quantum dots, J. Appl. Phys. 103, 034307 (2008).

[4] Y. Kawano, K. Ishibashi, An on-chip near-field terahertz probe and detector, Nature Photon 2, 618 (2008).

[5] M. Tonouchi, Cutting-edge terahertz technology, Nature Photonics 1, 97-105 (1997).

[6] S. E. M. Rinzan, G. Jenkins, H. D. Drew, S. Shafranjuk, and P. Barbara, Carbon Nanotube Quantum Dots As Highly Sensitive TerahertzCooled Spectrometers, Nano Lett. 12(6), 30973100 (2012); DOI: 10.1021/nl300975h.

[7] L. P. Kouwenhoven, , S. Jauhar, J. Orenstein, P. L. McEuen, Y. Nagamune, J. Motohisa, and H. Sakaki, Observation of photon assisted tunneling through a quantum dot. Phys. Rev. Lett. 73: 3443-3446 (1994).

[8] Shafraniuk, S. E. 2007. Sensing an electromagnetic field with photon-assisted Fano resonance in a two-branch carbon nanotube junction, Phys. Rev. B 76: 085317.

[9] S. E. Shafraniuk, Probing the intrinsic state of a one-dimensional quantum dot with a photonassisted tunneling, Phys. Rev B 78: 235115 (2008).

[10] Shafraniuk, S. E.. Electromagnetic properties of graphene junctions, European Physical Journal B 80: 379-393 (2011).

[11] S. E. Shafraniuk, Resonant transport through a carbon nanotube junction exposed to an ac field, Journal of Physics: Condensed Matter 23: 495304 (2011).

[12] S. Shafraniuk, Graphene and Carbon Nanotube Quantum Dot Sensors of the THz Waves, In: Multi Volume Set 'Nanotechnology', Studium Press LLC, USA, edited by S. Sinha and N. K. Navani, Vol. 10, Nanosensing (2012). 